\title{
State Estimation for Force-Controlled Humanoid Balance using Simple Models in the Presence of Modeling Error
}

\author{
Benjamin J. Stephens
}

\begin{abstract}
This paper considers the design of state estimators for dynamic balancing systems using a Linear Inverted Pendulum model with unknown modeling errors such as a center of mass measurement offset or an external force. A variety of process and output models are constructed and compared. For a system containing modeling error, it is shown that a naive estimator (one that doesn't account for this error) will result in inaccurate state estimates. These state estimators are evaluated on a force-controlled humanoid robot for a sinusoidal swaying task and a forward push recovery task.
\end{abstract}

\section{INTRODUCTION}

The goal of this paper is to provide a practical exploration of the effects of modeling error and unknown forces on state estimation for dynamic balancing humanoid robots. These disturbances have the effect that the observed dynamics do not match the expected dynamics, a discrepancy that cannot be simply represented as process noise. State estimators, such as a Kalman filter, are complicated by this because they rely on a prediction step that uses the expected forward dynamics. Likewise, model-based control frameworks, such as model predictive control (MPC), use the expected dynamics to generate a control policy. This policy is also often in the form of a state feedback controller which requires estimates of the full state of the system. This paper will explore how certain modeling errors can be overcome, either through changes to the process model in the estimator or the addition of specific sensing capabilities.

Humanoid balance is often studied using simplified models. One of the simplest and most widely-used is the Linear Inverted Pendulum Model (LIPM) [1] which treats the body as a lumped mass, centered at the center of mass (COM), that only moves horizontally. These assumptions result in a simple linear model of the dynamics, opening up the possibility of using any number of modern linear control and estimation techniques. One of the most widely used applications of the LIPM has been walking trajectory optimization using preview control [2] [3], which outputs a COM trajectory that satisfies the constraints that the center of pressure (COP) be within the base of support.

In general, many implementations of balance control on humanoid robots rely on stiff position-controlled joints and perfect tracking of the desired COM trajectory. However, these methods tend to fail in the presence of un-modeled disturbances such as uneven ground. This paper is focused on robots with force-controlled joints, such as the Sarcos

B. J. Stephens is a member of the Robotics Institute, Carnegie Mellon University, 5000 Forbes Ave, Pittsburgh, PA, USA. bstephens@cmu.edu, http://www.cs.cmu.edu/'bstephe1

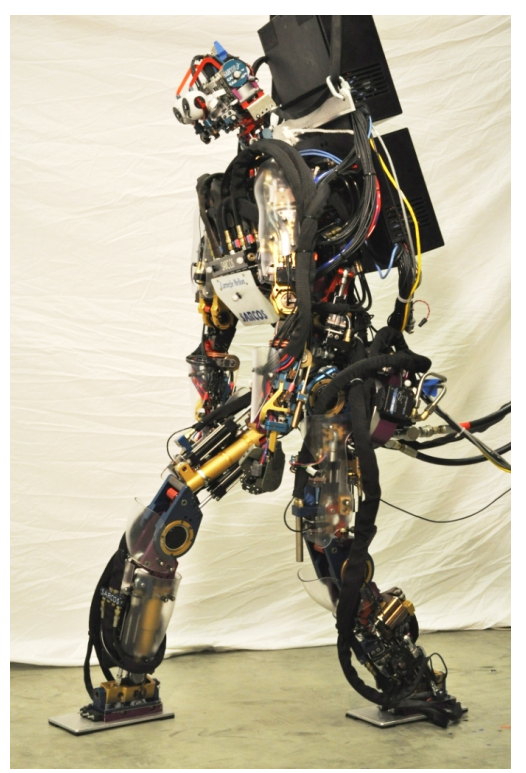

Fig. 1. The Sarcos Primus humanoid is a force-controlled robot that requires active balance control in the presence of modeling error.

Primus humanoid shown in Figure 1, that can comply to disturbances but require reactive balance control. Balance for force-controlled robots is often achieved using force-control techniques [4] [5] [6], where feedback is required to control COM state.

The problem being explored in this paper is that the state (position and velocity) of the COM are not easily determined. Not only is there no direct measurement of velocity, but modeling error can be significant. Often, modeling error has been modeled by augmenting the process and/or output models with step disturbances [7] [8]. The construction of these augmented models can have both positive and negative effects. This paper will consider both types of disturbances in the context of humanoid balance. First, an error in the true position of the COM offset can be represented by an output disturbance. Second, an unknown external force can be modeled by a state disturbance.

This paper is outlined as follows. In Section II, the different models for disturbances, sensors and estimators are presented and their performance is theoretically predicted by computing the observability of the system. Next, in Section III, state estimators are designed and compared for different cases of modeling error. Finally, in Section IV, the estimators are applied to the state estimation of a force-controlled humanoid robot performing different balance tasks. 


\section{SYSTEM MODELING}

For this paper, the 1D Linear Inverted Pendulum dynamics [1] are used as the base process dynamics.

$$
\ddot{x}=\omega^{2}\left(x-x_{c}\right)
$$

where $\omega=\sqrt{g / z_{0}}, x$ is the position of the center of mass (COM), $z_{0}$ is the constant height of the COM, and $x_{c}$ is the position of the center of pressure (COP). The LIPM dynamics are convenient because they can be written as a discrete linear system in statespace form,

$$
\mathbf{x}_{k+1}=\mathbf{A} \mathbf{x}_{k}+\mathbf{B} u_{k}
$$

where $\mathbf{x}_{k}=\left\{x, \dot{x}, x_{c}\right\}^{T}$ is the state vector and $u_{k}=\dot{x}_{c}$ is the input at timestep $k$. The matrices in (2) are given by

$$
\mathbf{A}_{0}=\left[\begin{array}{ccc}
1 & T & 0 \\
\omega^{2} T & 1 & -\omega^{2} T \\
0 & 0 & 1
\end{array}\right]
$$

and

$$
\mathbf{B}=\left[\begin{array}{l}
0 \\
0 \\
T
\end{array}\right]
$$

where $T$ is the timestep. This simple Euler approximation is used for clarity, although can be easily implemented for small $T$, such as the $T=0.0025 \mathrm{~s}$ corresponding to the $400 \mathrm{~Hz}$ control rate on the robot. $\mathbf{A}_{0}$ is the basic dynamics equation that will be used throughout this paper, though some modifications will be proposed.

A linear measurement model is also assumed to have the form,

$$
\mathbf{y}_{k}=\mathbf{C x}_{k}
$$

where $\mathbf{y}_{k}$ is a vector of measurements. The structure of C depends on the type of sensor modalities and state description.

\section{A. Sensing Models}

This section will describe various sensing models to to be used in (5). The sensor models are denoted by $\mathbf{C}_{i \mid j}$ where $i$ denotes the sensor model (i.e. $S_{i}$ ) and $j$ denotes the state dynamics (i.e. $A_{j}$ ). Combinations of sensor models will be represented by $\mathbf{C}_{i i \mid j}$, for example $\mathbf{C}_{12 \mid j}$.

S1) Position - Position sensors are the most common type of sensor. In this case, the position of the COM is calculated from a model of the mass distribution in the system. In general this mass distribution will be incorrect, so

$$
\mathbf{C}_{1 \mid 0}=\left[\begin{array}{lll}
1 & 0 & 0
\end{array}\right]
$$

S2) Center of Pressure - It is also common for balancing systems to have contact force sensors that measure the center of pressure of the system. In this case,

$$
\mathbf{C}_{2 \mid 0}=\left[\begin{array}{lll}
0 & 0 & 1
\end{array}\right]
$$

S3) Total COM Force - A ground contact force sensor can provide the total horizontal force on the COM. In this case, (1) shows up in the output model, multiplied by the mass of the system,

$$
\mathbf{C}_{3 \mid 0}=\left[\begin{array}{lll}
m \omega^{2} & 0 & -m \omega^{2}
\end{array}\right]
$$

\section{B. Modeling Errors}

Two general types common to humanoid robots are considered here: an unknown COM offset in the measurement output and an unknown external force. The modified process and output models for these will be presented.

D1) Unknown COM Offset - It is often the case in real systems that the true position of the COM will be unknown. This error can be represented by an output step disturbance,

$$
x_{k}=\hat{x}_{k}+\Delta x_{k}
$$

where $\hat{x}$ is the estimated COM position and $\Delta x$ is an unknown offset. In this case, a COM offset can be appended to the state vector, $\mathbf{x}=\left\{x, \dot{x}, x_{c}, \Delta x\right\}$ and re-write the process model as

$$
\mathbf{A}_{1}=\left[\begin{array}{cccc}
1 & T & 0 & 0 \\
\omega^{2} T & 1 & -\omega^{2} T & 0 \\
0 & 0 & 1 & 0 \\
0 & 0 & 0 & 1
\end{array}\right]
$$

which has the same dynamics as in (1).

D2) External Force - It also quite possible that an unmodeled external force could be perturbing the system. This force could represent an unknown contact with the world such as leaning against a wall, an interaction force such as shaking a hand, or an inertial frame force such as standing on a moving bus. In this case, the dynamics take the form,

$$
\ddot{x}=\omega^{2}\left(x-x_{c}\right)+u_{x}
$$

where $u_{x}$ is an unknown external force. In this case, an external force estimate can be appended to the process model, making the state, $\mathbf{x}=\left\{x, \dot{x}, x_{c}, u_{x}\right\}^{T}$, which has the dynamics of (11) and the state space dynamics,

$$
\mathbf{A}_{2}=\left[\begin{array}{cccc}
1 & T & 0 & 0 \\
\omega^{2} T & 1 & -\omega^{2} T & T \\
0 & 0 & 1 & 0 \\
0 & 0 & 0 & 1
\end{array}\right]
$$

D12) External Force and COM Offset - If both of the above modeling errors are assumed, then both an unknown external force and COM offset are appended to the dynamics of the system,

$$
\mathbf{A}_{12}=\left[\begin{array}{ccccc}
1 & T & 0 & 0 & 0 \\
\omega^{2} T & 1 & -\omega^{2} T & T & 0 \\
0 & 0 & 1 & 0 & 0 \\
0 & 0 & 0 & 1 & 0 \\
0 & 0 & 0 & 0 & 1
\end{array}\right]
$$

\section{State Estimators}

Process and measurement noise models are also constructed for the system to form the dynamics needed by the state estimator,

$$
\begin{aligned}
& \mathbf{x}_{k+1}=\mathbf{A} \mathbf{x}_{k}+\mathbf{B} \mathbf{u}_{k}+\nu_{x} \\
& \mathbf{y}_{k}=\mathbf{C x}_{k}+\nu_{y}
\end{aligned}
$$




\begin{tabular}{|l|c|c|}
\hline Variable & Process Noise & Output Noise \\
\hline Position & $1 \mathrm{e}^{-8}$ & $1 \mathrm{e}^{-5}$ \\
\hline Velocity & $1 \mathrm{e}^{-4}$ & \\
\hline COP & $1 \mathrm{e}^{-4}$ & $1 \mathrm{e}^{-5}$ \\
\hline COM Offset & $1 \mathrm{e}^{-4} / 1 \mathrm{e}^{-8}$ & \\
\hline External Force & $1 \mathrm{e}^{-4}$ & \\
\hline Totol Force & & 1 \\
\hline
\end{tabular}

Fig. 2. Table of covariance values used for the various estimators

where $\nu_{x}$ is a vector representing the process noise of each of the components of $\mathbf{x}_{k}$ and $\nu_{y}$ is a vector of measurement noise on each component of $\mathbf{y}_{k}$. The noise variables are assumed to be Gaussian processes with

$$
\begin{aligned}
& \nu_{x} \sim N(\mathbf{0}, \mathbf{Q}) \\
& \nu_{y} \sim N(\mathbf{0}, \mathbf{R})
\end{aligned}
$$

The covariance values used throughout this paper are shown in Figure 2.

An estimator will be denoted by $F_{i \mid j}$ which corresponds to a process model $\mathbf{A}_{j}$ and a sensor model $\mathbf{C}_{i \mid j}$. Below are descriptions of various estimator.

F0) Naive Estimator $\left(F_{i \mid 0}\right)$ - This estimator is referred to as naive because it has no representation of the modeling error.

F1) COM Offset Estimator $\left(F_{i \mid 1}\right)$ - For this estimator, the $A_{1}$ process model includes the COM offset as a variable and the ouput model is modified with a position measurement derived from (9),

$$
\mathbf{C}_{1 \mid 1}=\left[\begin{array}{llll}
1 & 0 & 0 & -1
\end{array}\right] \mathbf{x}
$$

F2) External Force Estimator $\left(F_{i \mid 2}\right)$ - In this case, the $A_{2}$ process model is used but the output model is unchanged, except for adding an additional column for the force, for example,

$$
\mathbf{C}_{12 \mid 1}=\left[\begin{array}{llll}
1 & 0 & 0 & 0 \\
0 & 0 & 1 & 0
\end{array}\right] \mathbf{x}
$$

F12) Dual Estimator $\left(F_{i \mid 12}\right)$ - In this case, the $A_{12}$ process model is used and the output model includes two additional columns, for example

$$
\mathbf{C}_{12 \mid 12}=\left[\begin{array}{ccccc}
1 & 0 & 0 & 0 & -1 \\
0 & 0 & 1 & 0 & 0
\end{array}\right] \mathbf{x}
$$

\section{Observability}

One way to partially predict the performance of different estimators is to consider the observability of the system defined by (14) and (15).

Given an estimator, $F_{i \mid j}$, observability is determined by evaluating [9]

$$
O\left(F_{i \mid j}\right)=\operatorname{rank}\left(\left[\begin{array}{c}
\mathbf{C}_{i \mid j} \\
\mathbf{C}_{i \mid j} \mathbf{A}_{j} \\
\mathbf{C}_{i \mid j} \mathbf{A}_{j}^{2} \\
\vdots \\
\mathbf{C}_{i \mid j} \mathbf{A}_{j}^{n-1}
\end{array}\right]\right)
$$

\begin{tabular}{|l|l|l|l|}
\hline Process Model & \multicolumn{3}{|c|}{ Sensor Model (Observability rank) } \\
\hline (state size) & S1 & S12 & S123 \\
\hline$A_{0}(n=3)$ (Naïve) & 3 & 3 & 3 \\
\hline$A_{1}(n=4)$ & $3(X)$ & 4 & 4 \\
\hline$A_{2}(n=4)$ & $3(X)$ & 4 & 4 \\
\hline$A_{12}(n=5)$ & $3(X)$ & $4(X)$ & $4(X)$ \\
\hline
\end{tabular}

Fig. 3. Table of observability given different process and output models. If the observability is equal to the size of the state vector then the state is fully observable. An (X) signifies that the system is not observable given these conditions.

where $n$ is the dimension of the state vector (including any offset parameters). If $O\left(F_{i \mid j}\right)=n$, then the system is observable. The table in Figure 3 gives this value for several different process and sensor model pairs.

Note that the dual estimator (F12) is not observable even given all three sensors (S123). This is because the S3 sensor does not provide an independent measure of the dynamics.

\section{STATE ESTIMATOR COMPARISONS}

In this section, the performance of various estimators in the presence of different modeling errors is compared. The estimators take as inputs data from known ground truth state trajectories generated by sinusoids with an amplitude, $a$, and frequency of $\beta$.

The state trajectories used have the form

$$
\begin{aligned}
x(t) & =a \sin \beta t \\
\dot{x}(t) & =a \beta \cos \omega t \\
x_{c}(t) & =x(t)+\frac{1}{\omega^{2}}\left(a \beta^{2} \sin \beta t+u_{x}\right)
\end{aligned}
$$

and COM measurements of

$$
\begin{aligned}
& y_{1}=x(t)-\Delta x \\
& y_{2}=x_{c}(t)
\end{aligned}
$$

\section{A. Naive Estimation}

First the need for estimators which account for modeling error is demonstrated. In this section a naive state estimator (F0) is implemented. However, when generating the ground truth trajectories, modeling error in the form of a COM measurement offset (D1) or an external force (D2) is added. The performance using position-only (S1) and position-COP (S12) measurement models is compared.

Figures (4) and (5) show the results using the naive estimators in the presence of a COM offset and an unknown external force, respectively. Using $\mathrm{S} 1$, the velocity is correctly estimated but results in a steady state offset of the COP. For the S12 case, the COP is correctly estimated but there is a steady state offset in the velocity. Both S1 and S12 fail in the D1 case to correctly estimate the COM position.

Because of this, it is desirable to modify the estimator to include additional states that correct for these steady state offsets. 

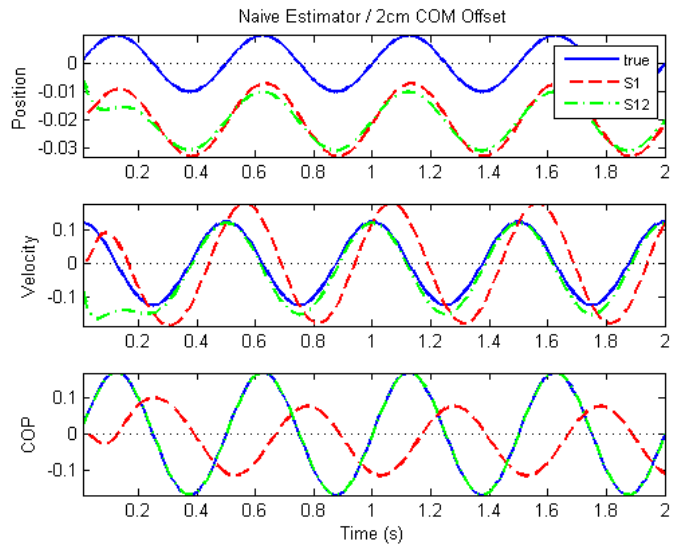

Fig. 4. State estimation performance unknown COM offset (D1) comparing position-only measurement (S1) with position and COP measurement (S12). A COM offset of $2 \mathrm{~cm}$ was used.
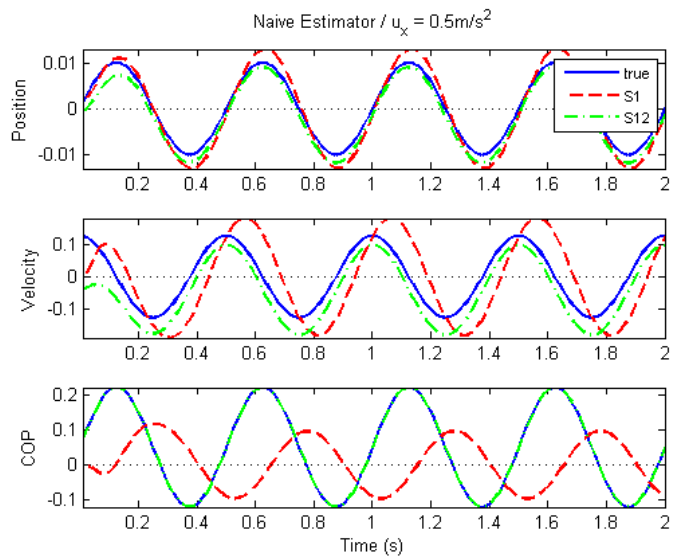

Fig. 5. State estimation performance with unknown external force (D2) comparing position-only measurement (S1) with position and COP measurement (S12). The external force was set such that $u_{x}=0.5$.

\section{B. COM Offset Estimation (F1)}

Results using a $F_{i \mid 1}$ state estimator in the presence of an output error of $\Delta x=2 \mathrm{~cm}$ are shown in Figure 6. Using only $\mathrm{S} 1$, the system is unable to estimate the state properly, whereas using S12 the estimator quickly converges.

\section{External Force Estimation (F2)}

Results using a $F_{i \mid 2}$ state estimator in the presence of a state disturbance of $u_{x}=0.5 \mathrm{~m} / \mathrm{s}^{2}$ are shown in Figure 7. Both $\mathrm{S} 1$ and $\mathrm{S} 12$ are able to estimate the COM position and velocity, but S12 is also able to estimate correctly the COP and the external force.

\section{EXPERIMENTAL RESULTS}

The intended application of these state estimators is to better estimate the COM state of the Sarcos Primus humanoid robot shown in Figure 1. It can be expected that both types of modeling error (D1 and D2) will be present in the system. Because of the complexity and high dimensionality of the robot, an offset in the modeled COM and the true COM is likely. In addition, the hydraulic hoses that provide the


Fig. 6. State estimation performance with unknown COM offset (D1) comparing position-only measurement (S1) with position and COP measurement (S12). The COM offset (with a true value of $\Delta x=0.02 m$ ) is added as an additional state variable in the process model.

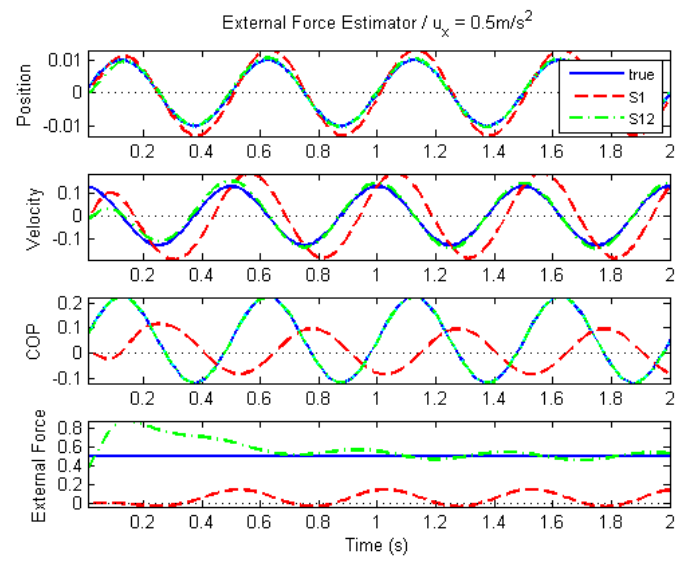

Fig. 7. State estimation performance with unknown external force (D2) comparing position-only measurement (S1) with position and COP measurement (S12). The external force (with a true value of $u_{x}=0.5 \mathrm{~m} / \mathrm{s}^{2}$ ) is added as an additional state variable in the process model.

the offboard power to the robot are known to apply external forces to the robot that cannot be measured. Below, the model of the robot used and its relation to the simplified models presented earlier is shown. Then the experimental setups are described and results are shown.

\section{A. Robot Model}

A rigid body dynamics model of the robot is assumed. The model has 12 actuated joints (6 in each leg) and a free-floating root joint (the hip). The torso and upper body joints are assumed to be rigidly attached to the hip and are controlled using stiff PD control with constant desireds.

From the robot, the measurements received are joint angles, q, from potentiometers, and hip orientation, $\mathbf{r}_{0}$, given by an IMU mounted to the hip. This leaves the hip position variables, $\mathbf{x}_{0}$, which are not observable. However, by assuming that a foot is flat on the ground at a known position these variables can be determined from the forward kinematics. There are two important features computed by 


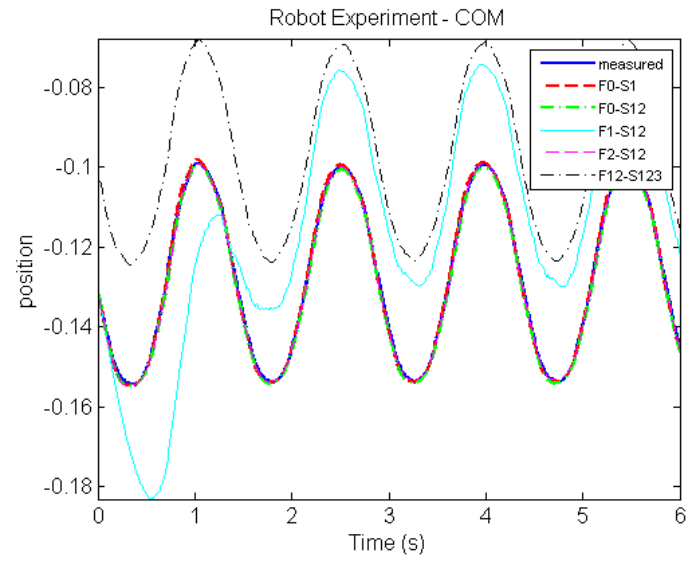

Fig. 8. Comparing the COM position estimation of multiple estimators, two of which estimate COM offset errors

the forward kinematics, including the foot centers,

$$
\begin{aligned}
& x_{L}=f_{L}\left(\mathbf{0}, \mathbf{r}_{0}, \mathbf{q}\right) \\
& x_{R}=f_{R}\left(\mathbf{0}, \mathbf{r}_{0}, \mathbf{q}\right)
\end{aligned}
$$

and the COM position,

$$
x=f_{C}\left(\mathbf{0}, \mathbf{r}_{\mathbf{0}}, \mathbf{q}\right)
$$

where $f_{X}(\mathbf{x}, \mathbf{r}, \mathbf{q})$ represents a forward kinematics function. In the above equations, $\mathbf{x}_{0}$ is set to zero. Therefore, assuming for example that the left foot is flat on the ground, the COM measurement is given by

$$
\hat{x}=x-x_{L}
$$

The COP measurement is computed by combining the measured COP under each foot using a 6-axis force/torque sensor. If $x_{p L}$ and $x_{p R}$ are the COPs and $F_{z L}$ and $F_{z R}$ are the vertical forces under the left and right foot, respectively, then

$$
\hat{x}_{c}=\frac{\left(x_{L}+x_{p L}\right) F_{z L}+\left(x_{R}+x_{p R}\right) F_{z R}}{F_{z L}+F_{z R}}-x_{L}
$$

if the total COP, again relative to the left foot.

\section{B. Experiments}

Two types of experiments were performed on the Sarcos Primus humanoid robot. In both experiments, the robot data was generated from a fixed balance controller using only low-pass filtered COM measurements and no state estimators. The state estimators were applied to this data and performance was compared. For verification, the robot was standing on a force-measuring platform. Though it is predicted to fail, a $F_{123 \mid 12}$ estimator is included for comparison which uses the force measurements from the force platform and the output model in (8). The lower covariance for the COM offset is used for the $F_{123 \mid 12}$ estimator because this variable is likely to change much slower than the external force.

Coronal Swaying - For this experiment, the robot is standing on a force-measuring platform and swaying side to

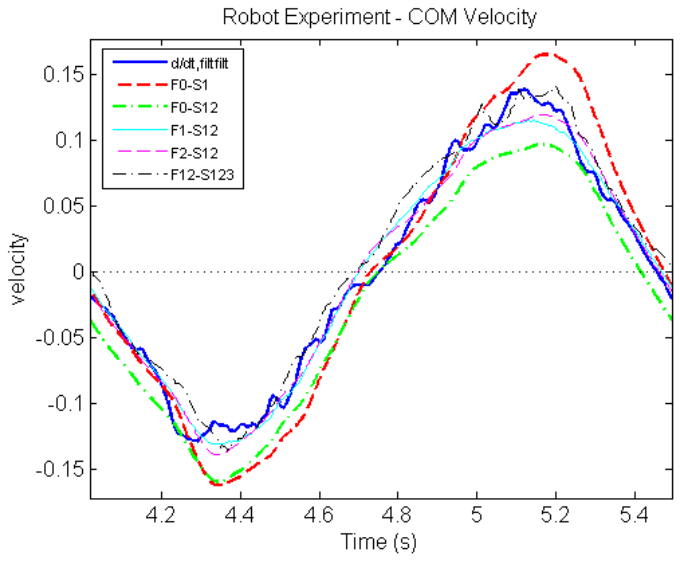

Fig. 9. Zoomed in view of the COM velocity estimation for multiple estimators

\begin{tabular}{|l|c|}
\hline Filter & RMS Velocity Error \\
\hline$F_{1 \mid 0}$ & 0.0055 \\
\hline$F_{12 \mid 0}$ & 0.0231 \\
\hline$F_{12 \mid 1}$ & 0.0015 \\
\hline$F_{12 \mid 2}$ & 0.0011 \\
\hline$F_{123 \mid 12}$ & 0.0084 \\
\hline
\end{tabular}

Fig. 10. Velocity estimation error of multiple estimators compared to a computed velocity

side in the coronal plane in a sinusoidal pattern. Using the measured COM and COP positions, several state estimators were applied to the data. Position estimates are shown in Figure 8. All estimators perform similarly, with $F_{i \mid 1}$ and $F_{i \mid 12}$ additionally estimating a significant COM offset of approximately $2.4 \mathrm{~cm}$. Figure 9 shows a zoomed-in view of the velocity estimates after some time has passed. The velocities are compared to a computed velocity that is calculated by differentiating and applying a zero-phase lowpass filter. The velocity estimation is generally improved by the more advanced estimators. The table in Figure 10 shows compares the error between the estimates and the computed velocity.

Sagittal Pushes - For this experiment, the robot is standing on a force-measuring platform and pushed from the front in the sagittal plane. The push is performed using a stick with a 6-axis force/torque sensor mounted at the end point. There are actually two types of external forces in this experiment. There is a small, nearly-constant external force caused by the hydraulic hoses in addition to the large momentary pushes from the experimenter. The F2 estimator was applied to this data with varying covariances for the external force variable and the results are shown in Figure 11. As the process noise covariance is increased, the estimator more closely estimates the push force, but also begins to incorrectly estimate external forces during the transient recovery period between pushes. 


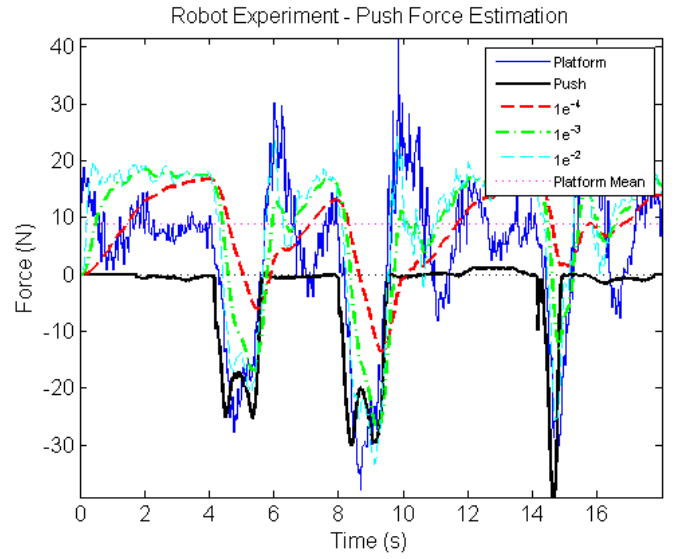

Fig. 11. External force estimation while undergoing pushes in the sagittal plane for various process noise models

\section{DISCUSSION AND FUTURE WORK}

This paper has presented through several examples, from both simulation and hardware, motivational evidence for the use of disturbance-modeling state estimators on forcecontrolled balance systems. Some of the key insights gained are summarized:

- Naive estimation in the presence of modeling error results in inaccurate results

- COM position only sensing (S1) almost always performs worse than when combined with COP sensing (S12)

- The presented models will not allow the estimation of both COM offset and external force disturbances (D12) simultaneously

- There is a trade-off between precisely estimating the disturbance and the state

The disturbance-modeling estimators presented use parametric models of the modeling error. However, the COM offset and external force disturbances are lumped-mass generalizations of real errors in the highly complex full-body robot. For example, perhaps there are small modeling errors in the COM position of every body in the rigid body dynamics model of the robot. Active estimation of a COM offset allows for online correction of these small errors without explicitly relying on the full model. This is the major motivation for applying simple models such as the LIPM to humanoid robots.

One of the limitations, however, of the LIPM is that it does not model the effects of angular momentum and external torques on the system. Models have been suggested to model these effects, often through the addition of a flywheel with a rotational inertia to the LIPM model. In this case the dynamics become

$$
\begin{aligned}
\ddot{x} & =\omega^{2}\left(x-x_{c}\right)-\frac{\tau}{m h} \\
I \ddot{\theta} & =\tau
\end{aligned}
$$

where a torque on the flywheel accelerates both the flywheel and the COM. The angle of the flywheel, $\theta$, can be roughly approximated by the angle of the torso. The added inertia increases the size of perturbations from which balance can be recovered [10].

Finally, time varying disturbances were only addressed briefly in Section IV. There are two approaches to handling this special class of disturbances. The first approach, suggested here, is careful tuning of the process model to change the rate at which disturbances are estimated. If the rate is too high, it is likely the system could go unstable. Another approach is to assume parametric models of the disturbances. If system expects to experience sinusoidal disturbances, for example if it were standing on boat, it might be worthwhile to model the disturbances in that way instead of a constant.

\section{CONCLUSION}

This paper presented the theory and design of state estimators that perform state estimation in the presence of modeling error by considering different disturbance models and sensor modalities. A variety of process and output models are constructed and compared. For a system containing modeling error, it is shown that a naive estimator (one that doesn't account for this error) will result in inaccurate state estimates. Finally, estimators are applied to the state estimation on a force-controlled humanoid robot for a sinusoidal swaying task and a push recovery task.

\section{ACKNOWLEDGEMENTS}

This material is based upon work supported in part by the National Science Foundation under grants DGE-0333420, ECCS-0325383, EEC-0540865, ECCS-0824077, and IIS0964581.

\section{REFERENCES}

[1] S. Kajita and K. Tani, "Study of dynamic biped locomotion on rugged terrain-derivation andapplication of the linear inverted pendulum mode," in Proceedings of the IEEE International Conference on Robotics and Automation, vol. 2, Apr. 1991, pp. 1405-1411.

[2] S. Kajita, F. Kanehiro, K. Kaneko, K. Fujiwara, K. Harada, K. Yokoi, and H. Hirukawa, "Biped Walking Pattern Generation by using Preview Control of Zero-Moment Point," in Proceedings fo the IEEE International Conference on Robotics and Automation, Taipei, Taiwan, Sep. 2003, pp. 1620-1626.

[3] P.-B. Wieber, "Trajectory Free Linear Model Predictive Control for Stable Walking in the Presence of Strong Perturbations," in Proceedings of the IEEE-RAS International Conference on Humanoid Robots, vol. 25 , no. 4 , 2006, pp. 137-142.

[4] J. Pratt, P. Dilworth, and G. Pratt, "Virtual model control of a bipedal walking robot," Proceedings of the IEEE International Conference on Robotics and Automation, vol. 1, pp. 193-198, Apr. 1997.

[5] S.-H. Hyon, J. G. Hale, and G. Cheng, "Full-Body Compliant Human Humanoid Interaction: Balancing in the Presence of Unknown External Forces," IEEE Transactions on Robotics, vol. 23, pp. 884-898, Oct. 2007.

[6] B. J. Stephens and C. G. Atkeson, "Dynamic Balance Force Control for Compliant Humanoid Robots," in Proceedings of the International Conference on Intelligent Robots and Systems, 2010.

[7] K. Muske and T. Badgwell, "Disturbance modeling for offset-free linear model predictive control," Journal of Process Control, vol. 12, no. 5, pp. 617-632, Aug. 2002.

[8] G. Pannocchia and J. B. Rawlings, "Disturbance models for offset-free model-predictive control," AIChE Journal, vol. 49, no. 2, pp. 426-437, Feb. 2003.

[9] C. I. Byrnes and C. F. Martin, "Global observability and detectability: An overview," Modelling and Adaptive Control, 1988.

[10] B. Stephens, "Humanoid Push Recovery," in Proceedings of the IEEERAS International Conference on Humanoid Robots, 2007. 